Orthopäde 2016 · 45:643

DOI 10.1007/s00132-016-3296-z

Online publiziert: 23. Juni 2016

๑) Springer-Verlag Berlin Heidelberg 2016

CrossMark

Die Inzidenz der Hüftdysplasie wird für Mitteleuropa mit 2-4\% angegeben. Die Rate der Hüftluxationen liegt bei etwa 0,5-1 \%. Das Verhältnis Mädchen zu Jungen beträgt ca. 4 zu 1. Das seit den 1990erJahren in Deutschland im Rahmen der U3 Untersuchung durchgeführte Sonographiescreening der Hüftgelenke hat da$\mathrm{zu}$ beigetragen, Hüftdysplasien frühzeitiger zu erkennen und zu behandeln. Die Rate der Folgeschäden bei Hüftdysplasien konnte deutlich gesenkt werden. Dennoch fallen immer wieder Kinder durch dieses Raster. Hinzu kommen Hüftdysplasien und Fehlstellungen des proximalen Femurs bei Patienten aus Ländern, in denen dieses Screening nicht regelhaft durchgeführt wird oder die eine syndromale bzw. neurologische Grunderkrankung aufweisen.

Die dauerhafte mechanische Überlastung des Hüftgelenks aufgrund einer reduzierten Kontaktfläche begünstigt die Entstehung einer vorzeitigen Arthrose. Diese Arthrosen des Kindes- und jungen Erwachsenenalters stellen die Patienten und die behandelnden Ärzte gleichermaßen vor große Herausforderungen. Gelenkerhaltende Eingriffe stoppen bei guter Indikationsstellung den Progress. Bei Dysplasiecoxarthrosen muss die Indikationsstellung sehr genau abgewogen werden. Eine Hüftdysplasie kann die jungen Patienten mit ihrem häufig hohen Aktivitätsbedürfnis erheblich einschränken. Im jungen Erwachsenenalter entstehende Arthrosen erfordern oft den frühzeitigen endoprothetischen Gelenkersatz, welcher in der Konsequenz langfristig Prothesenwechseloperationen mit den bekannten Risiken erwarten lässt. Insofern ist, neben einer frühzeitigen Diagnose, die frühe und adäquate Therapie der Hüftdysplasie und der Deformitäten des proximalen Femurs unerlässlich.

\author{
C. Lüring ${ }^{1} \cdot$ M. Tingart $^{2} \cdot$ A. Zahedi ${ }^{1} \cdot$ A. Schulze \\ 'Orthopädische Klinik, Klinikum Dortmund gGgmbH, Dortmund, Deutschland \\ ${ }^{2}$ Klinik für Orthopädie, Uniklinikum RWTH Aachen, Aachen, Deutschland
}

\title{
Osteotomien rund ums Becken
}

Stehen im Säuglingsalter aufgrund des hohen Nachreifungspotenzials der Hüftgelenke Schienenbehandlungen im Vordergrund, so stellen operative, die knöcherne Pathologie des Beckens und proximalem Femurs adressierende Verfahren, die Methode der Wahl dar, um einem vorzeitigen Gelenkverschleiß vorzubeugen.

Verschiedene Experten teilen in diesem Themenheft ihre Erfahrungen mit der Leserschaft und liefern wertvolle Praxistipps. Die etablierten und aktualisierten Therapiestrategien bei dysplastischen Deformitäten des Beckens und proximalen Femurs werden beschrieben und die Literaturergebnisse werden präsentiert und diskutiert.

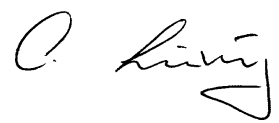

Prof. Dr. med. Christian Lüring

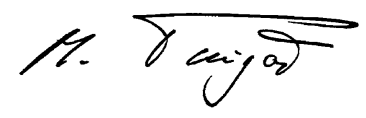

Univ.-Prof. Dr. med. Markus Tingart

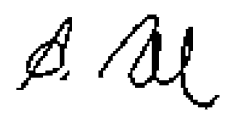

Dr. med. André Zahedi

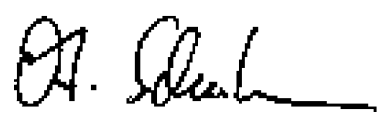

Dr. med. Astrid Schulze

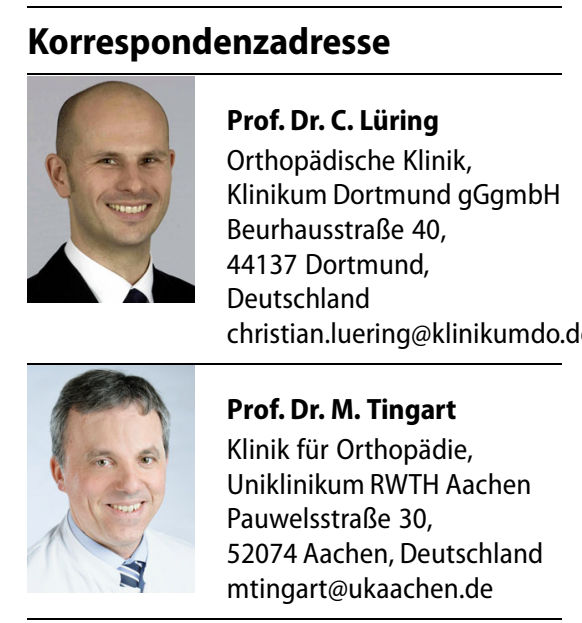

Interessenkonflikt. C. Lüring, M. Tingart, A. Zahedi und A. Schulze geben an, dass kein Interessenkonflikt besteht. 